\title{
Cross-sectional association between medical expenses and intellectual activity in community-dwelling older adults
}

Kimiko Tomioka* (D), Norio Kurumatani and Hiroshi Hosoi

\begin{abstract}
Background: Little is known concerning the lifestyle habits and health conditions in community-dwelling elderly who do not get medical care. We investigated the cross-sectional association between medical expenses (ME) and intellectual activity (IA) in community-dwelling older Japanese.

Methods: Self-administered questionnaires were mailed to all residents born between 1945 and 1949 and covered by A City's medical insurance system $(n=19,354)$. Independent variables including health behaviors, oral health, social capital, neighborhood environment, and physical and mental functioning were included in the questionnaires. Medical fee receipts were used to evaluate ME for fiscal 2014, and respondents were classified into no, low, medium, and high ME groups. Higher-level functional capacity was evaluated using the Tokyo Metropolitan Institute of Gerontology Index of Competence, which is comprised of three subscales: instrumental activities of daily living, IA, and social role. Poisson regression models were used to examine the association of ME with IA, with the low ME group as reference.
\end{abstract}

Results: Questionnaires were returned by 12,747 individuals (response rate 65.9\%). The no ME group had the lowest response rate, the worst lifestyle behaviors, and the lowest social capital, but no problems with neighborhood environment. Higher-level functional capacity, especially IA, was reduced in both the high ME and no ME groups. After adjustments for age, gender, health insurance, accessibility to public facilities in their residential area, family size, body mass index, and physical and mental functioning, the prevalence ratio (PR) for impaired IA lost its significance in the high ME group (PR 0.97, 95\% confidence interval 0.90-1.05), but remained significant in the no ME group (1.19, 1.08-1.31). After additional adjustments for health behaviors (i.e., health checks, smoking, fitness, and dietary variety), the PR of the no ME group was attenuated towards the null $(1.08,0.98-1.20)$.

Conclusions: Community-dwelling elderly who did not seek medical treatment were indifferent to health surveys and health-promoting behaviors, and undesirable health behaviors were a possible determinant of their impaired IA. Further longitudinal research is needed to confirm the causal associations.

Keywords: Community-dwelling elderly, Health behaviors, Higher-level functional capacity, Intellectual activity, Medical expenses

\footnotetext{
* Correspondence: tkimiko@naramed-u.ac.jp

Nara Prefectural Health Research Center, Nara Medical University, Shijo-cho

840, Kashihara city, Nara 634-8521, Japan
} 


\section{Background}

With the advancement of medicine and improvement of public health, average life expectancy has been increasing in both advanced countries and developing countries, meaning they must cope with societal aging. As of October 2015, the population aging rate in Japan is the world's highest, at $26.7 \%$ [1], and is proceeding at a much greater speed in Japan than in European and other western countries [1]. Ordinarily, elderly people tend to have many more ailments than younger people, so medical expenses (ME) naturally become much higher in aging populations [2]. In a super-aging society like Japan, the issue of suppressing health-care expenditures is urgent. Some municipalities in Japan have tested schemes such as giving a reward to those who do not receive health insurance treatment in an effort to suppress ME [3]. Therefore, it is necessary to investigate the lifestyle habits, health conditions, and higher-level functional capacities of the community-dwelling elderly who do not undergo medical treatment.

Higher-level functional capacity refers to a capacity that is beyond the basic activities of daily living (BADL) such as walking, having meals, taking a bath, and excreting. In other words, higher-level functional capacities are those required for elderly people to live independent lives in their community and include instrumental activities of daily living (IADL), intellectual activity (IA), and social role [4]. Maintaining these higher-level functional capacities signifies healthy aging. Past research on Japanese community-dwelling elderly has noted significant and independent associations between higher-level functional capacity and health behaviors such as smoking, fitness habits, and hours of sleep [5]; dietary habits such as dietary diversity [6] and animal protein intake [7]; oral health behaviors such as the use of extra cleaning devices [8]; oral function such as chewing ability [9]; social capital such as social participation [10]; and physical and mental functioning such as self-rated health [11], depression status [12], and cognitive performance [13]. On the other hand, significant factors involving increased ME include cardiovascular risk factors [14], obesity [15, 16], and less walking [17] among Japanese local residents; dietary diversity among Taiwanese elderly [18]; and meat consumption among Europeans and North Americans [19]. However, to the best of our knowledge, the relationships of higher-level functional capacity and medical care utilization on local-dwelling elderly are not known. To extend healthy life expectancy and realize a sustainable social security system in a super-aging society, it is meaningful to investigate the association between the extent of medical care utilization and higher-level functional capacity.

The aim of this study was to examine the crosssectional relationship between ME and higher-level functional capacity in Japanese aged 65-70 years, using community-based data.

\section{Methods \\ Study population}

Data were obtained from the Nara Data Health Survey, a cross-sectional population-based survey conducted by A City in Nara prefecture. Nara prefecture is positioned roughly in the center of Japan and is a landlocked municipality surrounded by the prefectures of Osaka, Kyoto, Wakayama, and Mie. During and after a high-growth period, Nara prefecture has become a bedroom community for the cities of Osaka and Kyoto. According to census figures [20], the prefecture with the highest rate of employed persons who are working in other prefectures is Nara. In October 2015, questionnaires were distributed to all people who satisfied the following three conditions: (1) they lived in A City on March 31, 2015, (2) they were born between 1945 and 1949, and (3) they were covered by the National Insurance, medical insurance for later-stage elderly (younger elderly people can be covered if their basic activities of daily living are low due to suffering from certain disorders), or livelihood subsidies, and the coverage period by one of the above was 1 year or longer as of March 31, 2015. As of January 1 in 2015, these three insurance systems covered an estimated 65\% of all citizens born from 1945 to 1949.

\section{Medical expenses}

The total ME of each subject was calculated based on medical fee receipts for the 12 months from April 2014 through March 2015. The total ME for fiscal 2014 was defined as each subject's ME and expressed in Japanese yen; according to foreign exchange rates of April 1, 2016, 0.90 US dollars or 0.78 euro equaled 100 Japanese yen. Based on their ME, participants who submitted questionnaires were categorized into two groups: those who did not have ME (hereafter, "no ME group") and those who had ME. The ME of men was significantly higher than those of women (Mann-Whitney test, $p<0.001$ ), and the ME of older people (ages 68 to 70 years at the time of the survey) was significantly higher than that of younger people (ages 65 to 67 years at the time of the survey) (Mann-Whitney test, $p<0.001$ ). Study participants with ME were then categorized into four groups based on gender and age (males 65 to 67 years old, males 68 to 70 years old, females 65 to 67 years old, and females 68 to 70 years old), and each group was separated into tertiles based on ME. Study participants were then categorized into four groups: no ME, low ME, medium ME, and high ME. 


\section{Health behaviors}

Assessments of health checkups were based on medical fee receipts, and the participants were dichotomized into having health checkups or having no health checkups during the 2014 fiscal year. Lifestyle habits were assessed by a self-administered questionnaire. Smoking habit was defined as never-smoker, ex-smoker, or current smoker. Number of years of smoking was classified as zero, 1$14,15-29$, or $\geq 30$. Frequency of alcohol consumption was classified as none, social, occasional, or daily. Frequency of exercise (hours per week) was classified as $<1$, $1-2,3-4$, or $\geq 5$. Walking time (minutes per day) was classified as $<30,30-59$, or $\geq 60$ [21]. Questions about dietary habits were measured using the dietary variety score (range 0-10) [22]; a higher score indicates a higher level of dietary variety. The participants were classified into tertiles according to their dietary variety score: T1 (scores of 0-2) indicated low variety, T2 (scores of 3-4) indicated medium variety, and T3 (scores of 5-10) indicated high variety.

\section{Oral health}

Oral health was assessed from a self-administered questionnaire that was based on previous studies [8, 9, 2325] and consisted of two parts: dental health behaviors and oral function. For dental health behaviors, we assessed the use of extra cleaning devices such as interdental brushes or dental floss (yes or no), the frequency of daily tooth brushing (three or more times a day, twice a day, or less than twice a day), and the frequency of bedtime tooth brushing (daily, sometimes, or almost never). Assessment of oral function included having difficulty in chewing hard foods (yes or no), choking on tea or soup (yes or no), having dry mouth (yes or no), and the use of dentures (yes or no).

\section{Social capital}

To measure social capital, we consulted previous studies [26-28] and decided to evaluate social participation, social support, and social networks through a questionnaire. Social participation was categorized into six types: volunteer groups, sports groups, hobby groups, senior citizens' clubs, neighborhood community associations, and cultural groups. The number of groups in which respondents participated was classified into none, one, two, and three or more. Social support was measured by the question, "How many people do you have that you can talk to if you feel troubled?: nobody (none), one person, two to three persons, four or more persons." Social networking was measured by the question, "How often do you meet your friends and acquaintances?: nearly every day, several times per week, several times per month, several times per year, almost never (none)."

\section{Neighborhood environment}

Because a prior study suggested that environmental factors can either facilitate or hamper medical care utilization [29], we assessed neighborhood environment using the Japanese version of the International Physical Activity Questionnaire Environment Module (IPAQ-E) [30]. To evaluate neighborhood environment related to $\mathrm{ME}$, we used two items from IPAQ-E: access to public facilities including medical institutions and access to public transport.

\section{Physical and mental functioning}

Assessment of physical and mental functioning was done by the self-administered questionnaire. Individuals with impaired BADL were defined as those who reported needing help to perform any of five BADL items: eating, dressing, bathing, going to the toilet, and walking indoors. Self-rated health was assessed through the single questionnaire item, "How is your health in general? Is it very good, rather good, rather poor, or very poor?" and was dichotomized into good (very good/rather good) and poor (rather poor/very poor). Health-related quality of life (QOL) was assessed using the Japanese version of the 8-item Short-Form Health Survey (SF-8) [31]. The SF-8 measures eight domains of health status and can be used to calculate two summary scores: the Physical Health Component Score (PCS) and the Mental Health Component Score (MCS), with higher scores indicating better QOL. The participants were classified into tertiles according to their PCS/MCS: T1 indicated low PCS/ MCS, T2 indicated medium PCS/MCS, and T3 indicated high PCS/MCS. Sleep disturbance was evaluated using the Pittsburgh Sleep Quality Index (score range 0-21) [32], and those with a score of $>5.5$ were considered to have sleep disturbance issues. Depression was evaluated using the 5-item short form of the Geriatric Depression Scale (score range 0-5) [33], and those with a score of $\geq$ 2 were considered to have depression. Cognitive functioning was evaluated using the Cognitive Performance Scale (score range 0-6), and those with a score of $\geq 1$ were considered to have poor cognitive functioning [34].

\section{Higher-level functional capacity}

Higher-level functional capacity was assessed using the Tokyo Metropolitan Institute of Gerontology Index of Competence (TMIG-IC) [35]. The TMIG-IC was developed to measure higher-level competence on the basis of Lawton's hierarchical model of behavioral competence [4]; the first five questions are about IADL (scores 0-5), the subsequent four questions are about IA (scores 0-4), and the final four questions are about social role (scores 0-4). A higher score indicates a higher functional level of higher-level competence. The reliability and validity of the TMIG-IC have been established [36]. For total 
score (score range $0-13$ ), participants with a score of $\leq$ 11 were defined as having impaired higher-level functional capacity. This cutoff value was based on a previous report that the variation of 1 point for total score on the TMIG-IC was regarded as a possible measurement error [37]. For subscale scores, participants with a score of 1 or more below the respective full mark were defined as having impaired capacity, i.e., a score of $\leq 4$ for IADL and a score of $\leq 3$ for IA and social role [38]. Because social role has a conceptual configuration similar to social capital, which was one of the independent variables in this study [4], we thought that selecting social role as a dependent variable was not appropriate due to the problem of multicollinearity. Additionally, because prior studies have revealed that impaired IA can predict a decline in IADL in community-dwelling older people [10, $11,39]$, we considered that IA was a more effective indicator than IADL of higher-level functional capacity in the elderly. Therefore, we decided to adopt IA as our main outcome. The IA subscale is defined as "activity emanating from the motivation to explore" [4] and is composed of four questions [35]: "Are you able to fill out forms for your pension?," "Do you read newspapers?," "Do you read books or magazines?," and "Are you interested in news stories or programs dealing with health?"

\section{Covariates}

Age, gender, type of health insurance, accessibility to public facilities in residential area, family size, and body mass index (BMI) were collected as covariates. Information on age, gender, health insurance, and residential area was provided by the city hall, and family size, BMI, and individual-level accessibility to public facilities were collected from a questionnaire. Health insurance was categorized as National Insurance, medical insurance for later-stage elderly, or livelihood subsidies. Family size was categorized as one (i.e., living alone), two, three, or four or more persons. The BMI $\left(\mathrm{kg} / \mathrm{m}^{2}\right)$ was calculated as weight $(\mathrm{kg})$ divided by height squared $\left(\mathrm{m}^{2}\right)$ and categorized as normal $(18.5-<25.0)$, underweight $(<18.5)$, or obese $(\geq 25.0)$ based on the definition by the Japan Society for the Study of Obesity [40]. For accessibility to public facilities, area-level facilities instead of individuallevel facilities were set as covariates with the purpose of adjusting for local characteristics. In detail, A City was divided into 50 areas based on sphere of life, and the rate of participants who answered "poor" on accessibility to public facilities in each area was calculated based on accessibility to public facilities as evaluated with IPAQ-E [30]. The calculated rate of those who answered "poor" was then divided in two at the median value and classified into the areas of either "good" or "poor" based on accessibility to public facilities. As a result, 7421 residents in 25 areas were categorized as living in "good" areas, and 5326 residents in 25 areas were categorized as living in "poor" areas.

\section{Statistical analysis}

A trend test to detect an increased prevalence of participants' characteristics among the four ME groups was performed using the Cochran-Armitage test. To examine the relationship between $\mathrm{ME}$ as an independent variable and IA as a dependent variable, a prevalence ratio (PR) and a 95\% confidence interval (CI) for impaired IA (i.e., IA score $\leq 3$ ) were calculated using Poisson regression analyses with robust variance estimators [41]. Participants in the low ME group were set as the reference category. Age (continuous), gender, health insurance, accessibility to public facilities in their residential area, family size, and BMI were adjusted for in Model 1. In addition, self-rated health, health-related QOL (PCS and MCS), sleep disturbance, depression, and cognitive functioning (i.e., physical and mental functioning) were adjusted for in Model 2; BADL was not included in Model 2 because all people with medical insurance for laterstage elderly were limited in BADL. To investigate which factors explained impaired IA in the no ME group, health behaviors, oral health, or social capital were added to each model from Model 3 to Model 5, and changes in the PR estimates for impaired IA were explored. When the PR for impaired IA was attenuated towards the null and underwent a major change, it was considered that the additional factor was a more potential determinant of impaired IA in the no ME group than were other factors. Model 3 added participation in health checks, current smoking status, frequency of exercise, and dietary variety (i.e., health behaviors) to the variables in Model 2. Model 4 added the use of extra cleaning devices, the frequency of bedtime brushing, difficulty with hard foods, and the use of dentures (i.e., oral health) to the variables in Model 2; frequency of daily brushing was excluded from Model 4 because of a significant association with the frequency of bedtime brushing (Spearman's coefficient $=0.56$ ). Model 5 added social participation, social support, and social networks (i.e., social capital) to the variables in Model 2.

Statistical analyses were performed using SPSS version 24.0 J (IBM SPSS Inc., Chicago, USA) with a significance level of $5 \%$ (two-sided test).

\section{Results}

Among the 19,354 eligible individuals, 12,747 returned the questionnaires (response rate 65.9\%). Basic attributes of individuals with or without submission of the questionnaire are shown in Table 1 and Fig. 1. Compared to individuals who submitted the questionnaire, nonsubmitters were more likely to be male, young, and 
Table 1 Basic attributes of individuals with or without submission of the questionnaire

\begin{tabular}{|c|c|c|c|c|c|}
\hline \multirow[t]{2}{*}{ Basic attributes } & \multicolumn{2}{|c|}{ Submitters of the questionnaire $(n=12,747)$} & \multicolumn{2}{|c|}{ Non-submitters of the questionnaire $(n=6607)$} & \multirow[t]{2}{*}{$p^{a}$} \\
\hline & $n$ & $\%$ & $n$ & $\%$ & \\
\hline \multicolumn{6}{|l|}{ Gender } \\
\hline Male & 5413 & 42.5 & 3073 & 46.5 & \multirow[t]{2}{*}{$<0.001$} \\
\hline Female & 7334 & 57.5 & 3534 & 53.5 & \\
\hline \multicolumn{6}{|l|}{ Health insurance } \\
\hline National Insurance & 12,321 & 96.7 & 6295 & 95.3 & \multirow[t]{3}{*}{0.082} \\
\hline Medical insurance for later-stage elderly & 109 & 0.9 & 73 & 1.1 & \\
\hline Livelihood subsidies & 317 & 2.5 & 239 & 3.6 & \\
\hline \multicolumn{6}{|l|}{ Basic activities of daily living dependency ${ }^{b}$} \\
\hline Absent & 12,480 & 97.9 & 6340 & 96.0 & \multirow[t]{2}{*}{$<0.001$} \\
\hline Present & 267 & 2.1 & 267 & 4.0 & \\
\hline
\end{tabular}

${ }^{a}$ Differences between submitters and non-submitters were analyzed using the chi-square test

b Individuals who were certified as in need of support/care-level as per the national long-term care insurance system

dependent in BADL. Regarding ME, the response rate was lowest for residents who had no ME; the second lowest response rate was for those who had the highest ME.

Regarding the characteristics of the study population (see Additional file 1), males were more likely to be older, live with more persons, be obese, have health checkups, be smokers and daily drinkers, exercise, have difficulty in chewing hard foods, and suffer depression and poor cognitive functioning. Females were more likely to have National Insurance, be underweight, have high dietary variety, have good dental health behaviors, have good self-rated health and high MCS, suffer sleep disturbance, have a high level of social capital, and preserve higher-level functional capacity.

Table 2 shows the characteristics of the study population by four groups based on ME. Participants with more ME were more likely to be obese, have less time to walk, have more difficulty swallowing, have dry mouth, report poor physical and mental functioning, and perceive the environment of their neighborhood as poor. In contrast, the no ME group had the worst scores in health behaviors other than walking time, dental health behaviors, chewing ability, and social capital across the four groups. Additionally, the no ME group had the same level of impaired higher-level functional capacity as the high ME group.

The prevalence of impaired higher-level functional capacity by subscale was evaluated (see Additional file 2). Participants with impaired IADL were observed most frequently in the high ME group, while for IA and social role, those with impaired capacity were equally represented in the high ME group and the no ME group.
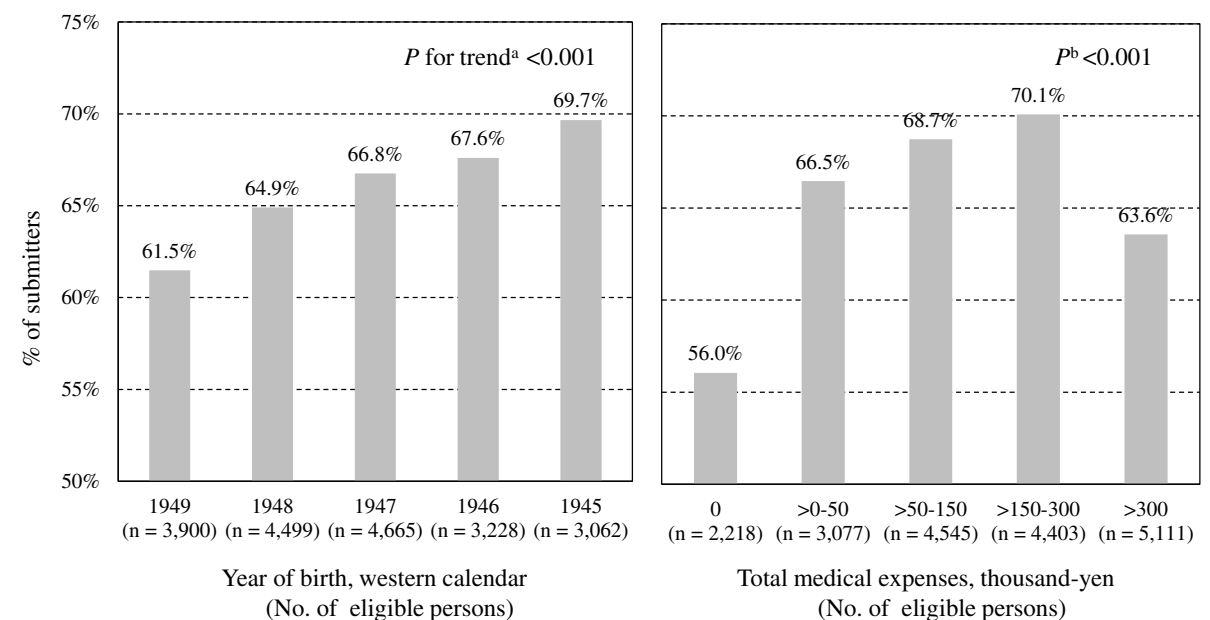

Fig. 1 Percentage of individuals who submitted the questionnaire by year of birth and total medical expenses for the 2015 fiscal year. Superscript "a" indicates Cochran-Armitage test, and superscript "b" indicates chi-square test 
Table $\mathbf{2}$ Characteristics of the study population by medical expense category

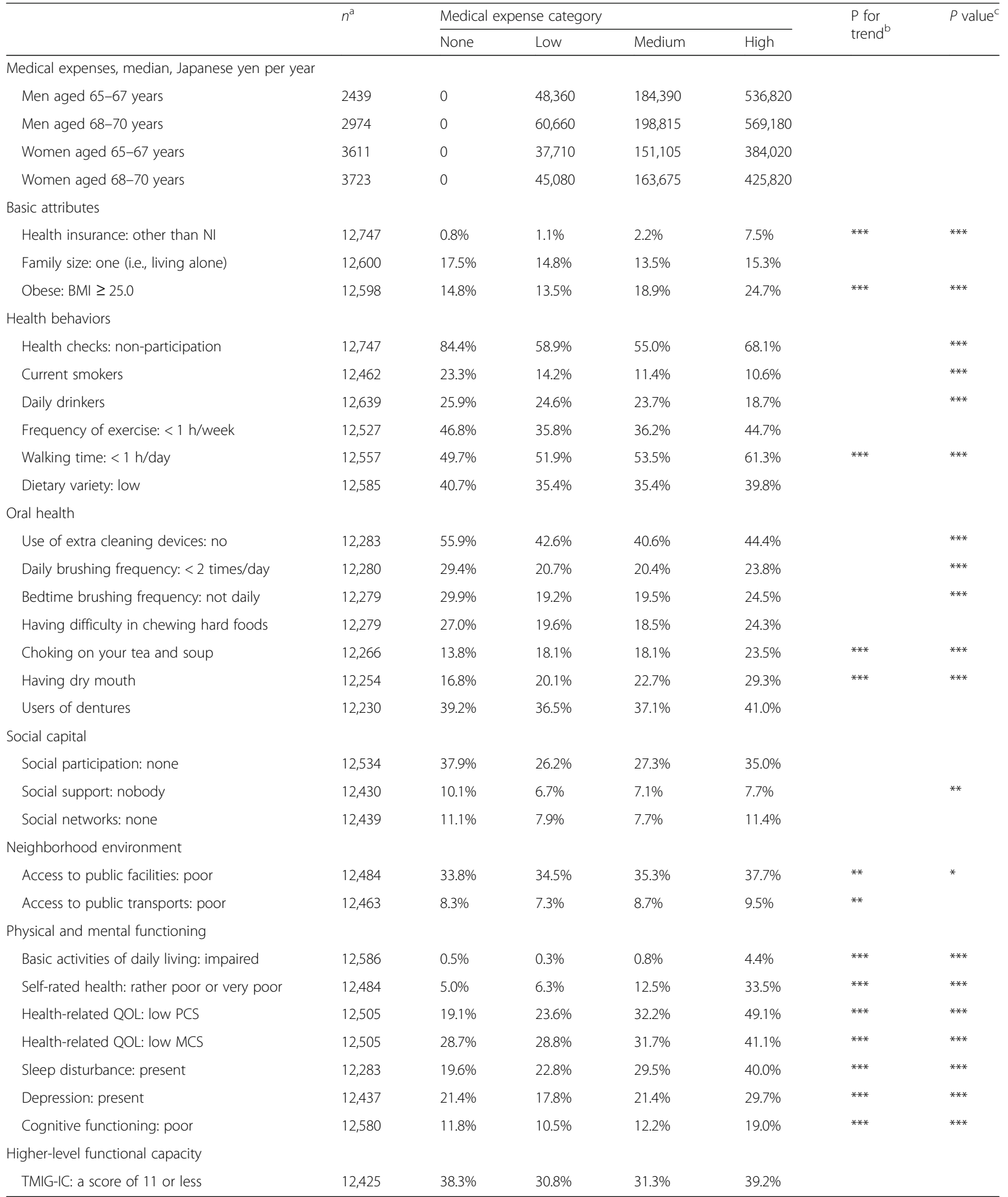

MCS Mental Component Summary, ME medical expenses, NI National Insurance, PCS Physical Component Summary, QOL quality of life, TMIG-IC Tokyo Metropolitan Institute of Gerontology Index of Competence

${ }^{*} p<0.05 ;{ }^{* *} p<0.01 ;{ }^{* * *} p<0.001$

${ }^{a}$ Number of valid responses

${ }^{b}$ Dose-response relationship between more ME and more persons corresponding to the item was analyzed using the Cochran-Armitage test

'Difference between the no ME group and the high ME group was analyzed using Fisher's exact test 
Table 3 shows the PRs for impaired IA associated with ME. In the crude model, the high ME group and the no ME group were significantly associated with impaired IA: $\mathrm{PR}=0.98(95 \% \mathrm{CI}=0.91-1.06)$ for the medium $\mathrm{ME}$ group, 1.26 (1.17-1.35) for the high ME group, and 1.27 (1.16-1.40) for the no ME group, compared to the low ME group. These significant associations were unchanged after adjustment for covariates (Model 1). However, after additional adjustment for physical and mental functioning (Model 2), the association of the high ME group with impaired IA disappeared, and a significant association remained only for the no ME group: $\mathrm{PR}=0.97$ (95\% CI $=0.90-1.05)$ for the high ME group and 1.19 (1.08-1.31) for the no ME group. To explore explanatory factors for impaired IA among the no ME group, we added health behaviors, oral health, or social capital to each model from Model 3 to Model 5. In Model 3, where the data were adjusted for health behaviors in addition to the variables in Model 2, the PR of the no ME group dropped to 1.08 (95\% CI $=0.98-1.20$ ), and significance disappeared. After adjusting for oral health (Model 4) or social capital (Model 5), the PRs of the no ME group were attenuated, but remained statistically significant. To check the effect of missing values in covariates and independent variables, we performed additional analyses that included missing data of each variable as a missing category. Although these re-analyses strengthened and partly altered the significant results, the main result that health behaviors had the greatest effect on impaired IA in the no ME group was unchanged (see Additional file 3).

Table 3 Prevalence ratios for impaired IA (IA score $\leq 3$ ) associated with medical expenses

\begin{tabular}{|c|c|c|c|c|c|c|c|}
\hline & $\begin{array}{l}\text { Prevalence of } \\
\text { impaired IA }\end{array}$ & $\begin{array}{l}\text { Crude } \\
(n=12,458) \\
\text { PR }(95 \% \text { Cl) }\end{array}$ & $\begin{array}{l}\text { Model } 1^{\text {a }} \\
(n=12,305) \\
\text { PR }(95 \% \text { Cl) }\end{array}$ & $\begin{array}{l}\text { Model } 2^{b} \\
(n=11,679) \\
\text { PR }(95 \% \text { Cl) }\end{array}$ & $\begin{array}{l}\text { Model } 3^{c} \\
(n=11,498) \\
\text { PR }(95 \% \text { Cl) }\end{array}$ & $\begin{array}{l}\text { Model } 4^{d} \\
(n=11,228) \\
\text { PR }(95 \% \text { Cl) }\end{array}$ & $\begin{array}{l}\text { Model } 5^{\mathrm{e}} \\
(n=11,318) \\
\text { PR }(95 \% \mathrm{Cl})\end{array}$ \\
\hline \multicolumn{8}{|l|}{ Medical expenses in the past year } \\
\hline Low & $25.5 \%$ & 1.00 & 1.00 & 1.00 & 1.00 & 1.00 & 1.00 \\
\hline Medium & $25.1 \%$ & $0.98(0.91-1.06)$ & $0.98(0.90-1.05)$ & $0.93(0.86-1.00)$ & $0.94(0.87-1.01)$ & $0.94(0.87-1.02)$ & $0.93(0.86-1.01)$ \\
\hline High & $32.1 \%$ & $1.26(1.17-1.35)$ & $1.16(1.08-1.25)$ & $0.97(0.90-1.05)$ & $0.99(0.91-1.07)$ & $0.96(0.89-1.04)$ & $0.98(0.90-1.06)$ \\
\hline None & $32.5 \%$ & $1.27(1.16-1.40)$ & $1.23(1.11-1.35)$ & $1.19(1.08-1.31)$ & $1.08(0.98-1.20)$ & $1.12(1.01-1.24)$ & $1.14(1.03-1.26)$ \\
\hline \multicolumn{8}{|l|}{ Health behaviors } \\
\hline Health checks: no (ref: yes) & & & & & $1.07(1.003-1.14)$ & & \\
\hline $\begin{array}{l}\text { Smoking: current } \\
\text { (ref: never/ex-smoker) }\end{array}$ & & & & & $1.28(1.20-1.38)$ & & \\
\hline $\begin{array}{l}\text { Frequency of exercise }(h / w) \text { : } \\
<1 \text { (ref: } \geq 1)\end{array}$ & & & & & $1.46(1.38-1.56)$ & & \\
\hline Dietary variety: low (ref: high) & & & & & $1.27(1.19-1.35)$ & & \\
\hline \multicolumn{8}{|l|}{ Oral health } \\
\hline $\begin{array}{l}\text { Use of extra cleaning devices: } \\
\text { no (ref: yes) }\end{array}$ & & & & & & $1.16(1.09-1.24)$ & \\
\hline $\begin{array}{l}\text { Bedtime brushing: not daily } \\
\text { (ref: daily) }\end{array}$ & & & & & & $1.17(1.10-1.26)$ & \\
\hline $\begin{array}{l}\text { Difficulty with chewing hard } \\
\text { foods: yes (ref: no) }\end{array}$ & & & & & & $1.10(1.02-1.17)$ & \\
\hline Use of dentures: yes (ref: no) & & & & & & $1.02(0.96-1.09)$ & \\
\hline \multicolumn{8}{|l|}{ Social capital } \\
\hline $\begin{array}{l}\text { Social participation: no } \\
\text { (ref: yes) }\end{array}$ & & & & & & & $1.44(1.35-1.53)$ \\
\hline Social support: no (ref: yes) & & & & & & & $1.19(1.10-1.29)$ \\
\hline Social networks: no (ref: yes) & & & & & & & $1.28(1.18-1.38)$ \\
\hline
\end{tabular}

PRs and $95 \%$ Cls were calculated using Poisson regression analyses

$C l$ confidence interval, $I A$ intellectual activity, ref. reference, $P R$ prevalence ratio

${ }^{a}$ Model 1 is adjusted for age, gender, health insurance, accessibility to public facilities in their residential area, family size, and body mass index

${ }^{\mathrm{b}}$ Model 2 is adjusted for the covariates in Model 1 plus physical and mental functioning (self-rated health, health-related QOL (PCS and MCS), sleep disturbance, depression, and cognitive functioning)

'Model 3 is adjusted for the variables in Model 2 plus health behaviors (health checks, smoking, frequency of exercise, and dietary variety)

${ }^{\mathrm{d}}$ Model 4 is adjusted for the variables in Model 2 plus oral health (use of extra cleaning devices, frequency of bedtime brushing, difficulty with chewing hard foods, and use of dentures)

eModel 5 is adjusted for the variables in Model 2 plus social capital (social participation, social support, and social network) 


\section{Discussion}

Our study was the first to examine the association between a variety of factors and ME and reveal characteristics of the no ME group among community-dwelling elderly Japanese. The findings that community-dwelling elderly with no ME were less willing to cooperate with the survey, rarely had physical checkups, generally smoked and drank more often, did less exercise, had lower dietary variety, and practiced poor dental health behaviors suggested that they are less interested in their health and have unhealthier lifestyle behaviors, which could include not visiting doctors. Prior studies demonstrated that ME was significantly increased among obese people $[15,16]$ and reduced among people who spent a longer time walking [17]. Similar findings were confirmed in our study population, but lifestyle habits, including oral hygiene, were worst in the no ME group, with the exception of walking. A Germany study of adults aged 35 years or older [42] reported that higher health check attendance was associated with nonsmoking, brisker physical activity, higher fruit and vegetable intake, and higher use of ambulatory care; not receiving health checks was thought to be a reflection of an unhealthy lifestyle choice, which supports our findings. Therefore, we propose that community-dwelling elderly with no ME require more intervention regarding their lifestyle habits.

We observed that the no ME group had the same degree of impaired higher-level functional capacity as the high ME group. An analysis of factors responsible for impaired IA suggested that impaired capacity in the high ME group could be explained by poor physical and mental functioning. The no ME group was high in physical and mental functioning but low in health-promoting behavior, and unhealthy lifestyle was identified as a possible determinant of impaired IA in this group. Many epidemiological studies have indicated that physical and mental functioning are strong contributors to higherlevel functional capacity of the elderly $[11-13,38,43$, 44]. In particular, low IA is strongly associated with sensory impairment $[38,43]$ and a low grade of cognitive functioning [44].

Our findings that the no ME group had good physical and mental conditions but poor IA and social role are inconsistent with previous studies. One possible explanation for low IA in the no ME group is that in the TMIG-IC, the IA subscale includes reading and interest in health-related information [35]. Participants who were included in the no ME group and did not have healthy daily habits were considered to have lower IA because they were not interested in obtaining information about health and had fewer opportunities to read newspapers, books, and magazines. Regarding the mechanism underlying the association between health behaviors and IA,
IA is a high-level life function represented by leisure activities, creativity, and searching [4,35], and evaluation items of IA are classified into cognitively stimulating activities [39, 44]. Bad daily habits like smoking, having poor nutritional condition from consuming a smaller variety of foods, and missing regular exercise are connected to decreased oxygen and nutrition in the brain, which may cause the deterioration in their cognitive activities [24, 45]. This hypothesis is supported by prior prospective studies that reported an association of higher dietary variety $[6,22]$ and higher animal protein [7] with a lower risk of IA decline in older communitydwelling adults. However, this hypothesis is inconsistent with another prior study [5] that reported a significant association of current smoking and low frequency of exercise with the risk of low social role, but not with a decline in IA.

Our results showed that self-rated health became worse as ME became higher, which agrees with the findings from a previous study that regular visits to medical doctors were a prime determinant of self-rated health for Japanese community-dwelling elderly [46]. Because the no ME group had good health conditions and did not perceive themselves as being unhealthy, they had no reason to see medical doctors. However, the no ME group also had unhealthy lifestyle habits; even if they were not conscious of their poor health, there was a strong likelihood that they could contract lifestylerelated diseases but leave them untreated. It is therefore highly probable that community-dwelling elderly with no ME will have substantially greater needs for future hospitalization and continuous health care. Additionally, deterioration in IA can increase the risk of cognitive decline [44] and incident disability in BADL [11], which makes long-term care insurance necessary. A further prospective study is expected to confirm whether community-dwelling elderly with no ME have a high risk of augmenting near-future social security costs.

In our study, response rate showed a linear increase with age (see Fig. 1). Although prior studies failed to collect data on non-respondents $[10,47,48]$, they suggested that younger people tended to respond to the questionnaire better, which is inconsistent with our results. A possible explanation for a lower response rate in younger age is that they are more involved in paid work than older people. Because working persons did not have enough free time, they may have been less likely to participate in our study than individuals without a job. Although we have no data on current working status, we think there is a high probability that job status may affect the response rate. Future studies are needed to verify whether the association between younger age and a lower response rate is observed in other age groups as well. 
This study had some limitations. First, because our study was cross-sectional, we cannot confirm causal relationships. To overcome this limitation, a further prospective study should be performed. Second, nearly half of the community-dwelling elderly with no ME did not participate in this survey. This is consistent with a prior study reporting that people who had no healthcare utilization had lower levels of involvement in health surveys than people who had made at least one visit to the doctor [49]. This may have resulted in selection bias and requires special attention in the interpretation of the results. It is well-known that people who cooperate with health surveys are more interested in health and have better health behaviors and conditions compared to non-cooperators [50]. Therefore, lifestyle habits and higher-level functional capacity among the no ME group may have been underestimated. Third, our study's sample was drawn from community-dwelling elderly aged 65-70 years. People of this generation were considered eligible because they could answer many questions more easily than people aged 70 years or older, and because there is a concern that they will have a major influence on social security costs in the near future. However, our findings on community-dwelling elderly above the age of 70 or elderly living in institutions should be interpreted with caution. Finally, we could not assess socioeconomic factors such as income and education, which have been shown to have effects on health care utilization [51], health behaviors [52], and IA [45]. Because the Japanese government offers health protection to economically poor persons through a welfare system, income may have an insignificant effect on physician visits, but education has the potential to be an important determinant of consultative behavior. Prior studies have demonstrated that lower educational attainment is associated with lower level of IA $[38,45]$. Because our findings have a potential for confounding by educational background, future studies should consider education when evaluating the association between ME and IA among community-dwelling elderly.

\section{Conclusions}

This cross-sectional study found that communitydwelling elderly with no ME had a low response rate, unhealthy lifestyle habits, and a low level of social capital. Higher-level functional capacity was diminished among both the high ME group and the no ME group. Our findings suggest that community-dwelling elderly who do not receive medical treatment were indifferent to health surveys and health-promoting behaviors, and undesirable health behaviors were a possible determinant of their impaired IA. However, further longitudinal studies are needed to explore the underlying causal relationships between ME, health behaviors, and IA among the community-dwelling elderly.

\section{Additional files}

Additional file 1: Table S1. Characteristics of the study population responding to the questionnaire $(n=12,747)$. (PDF $102 \mathrm{~kb}$ )

Additional file 2: Figure S1. Percentage of participants with impaired capacity in each subscale of higher-level functional capacity according to medical expenses. (PDF $50 \mathrm{~kb}$ )

Additional file 3: Table S2. Prevalence ratios for impaired IA (IA score $\leq 3)$ : Additional analyses with added missing data of each variable $(n=12,458)$. (PDF $72 \mathrm{~kb})$

\section{Abbreviations}

BADL: Basic activities of daily living; BMI: Body mass index; Cl: Confidence interval; IA: Intellectual activity; IADL: Instrumental activities of daily living; IPAQ-E: International Physical Activity Questionnaire Environment Module; MCS: Mental Health Component Score; ME: Medical expenses; PCS: Physical Health Component Score; PR: Prevalence ratio; QOL: Quality of life; SF-8: 8item Short-Form Health Survey; TMIG-IC: Tokyo Metropolitan Institute of Gerontology Index of Competence

\section{Acknowledgments}

The authors wish to express our gratitude to all the participants for their cooperation in this study.

Funding

This work was supported by the Center Administrative Expense from Nara Medical University.

Availability of data and materials

All data generated or analyzed during this study are included in this published article and its supplementary information files.

\section{Authors' contribution}

$\mathrm{KT}$ provided the data collection and the analysis and interpretation of data, performed the statistical analyses, and drafted this manuscript. $\mathrm{NK}$ and $\mathrm{HH}$ provided the concept and design of the study and provided intellectual input into the article. All authors read and approved the final manuscript.

\section{Ethics approval and consent to participate}

This study protocol was approved by the Nara Medical University Ethics Committee (approval number 1118). All study participants provided written informed consent.

Consent for publication

Not applicable.

Competing interests

The authors declare that they have no competing interests.

\section{Publisher's Note}

Springer Nature remains neutral with regard to jurisdictional claims in published maps and institutional affiliations.

Received: 26 June 2017 Accepted: 17 August 2017

Published online: 25 August 2017

References

1. Cabinet Office. Government of Japan. Annual Report on the Aging Society. 2015; Available from: http://www8.cao.go.jp/kourei/whitepaper/index-w. html. Accessed 14 Aug 2017

2. Bloom DE, Chatterij S, Kowal P, Lloyd-Sherlock P, McKee M, Rechel B, et al. Macroeconomic implications of population ageing and selected policy responses. Lancet. 2015;385:649-57.

3. Soja City. Healthy and 10,000 yen cash back. Available from: http://www.city.soja. okayama.jp/kenkouiryou/iryou_fukushi/cashback.html. Accessed 14 Aug 2017.

4. Lawton MP. Assessing the competence of older people. In: Kent DP, Kastenbaum R, Sherwood S, editors. Research planning and action for the elderly: the power and potential of social science. New York: Behavioral Publications; 1972. p. 122-43. 
5. Tsubota-Utsugi M, Ito-Sato R, Ohkubo T, Kikuya M, Asayama K, Metoki H, et al. Health behaviors as predictors for declines in higher-level functional capacity in older adults: the Ohasama study. J Am Geriatr Soc. 2011;59:1993-2000.

6. Otsuka R, Kato $Y$, Nishita $Y$, Tange C, Nakamoto M, Tomida M, et al. Dietary diversity and 14-year decline in higher-level functional capacity among middle-aged and elderly Japanese. Nutrition. 2016;32:784-9.

7. Imai E, Tsubota-Utsugi M, Kikuya M, Satoh M, Inoue R, Hosaka M, et al. Animal protein intake is associated with higher-level functional capacity in elderly adults: the Ohasama study. J Am Geriatr Soc. 2014;62:426-34.

8. Moriya S, Tei K, Yamazaki Y, Hata H, Kitagawa Y, Inoue N, et al. Relationships between higher-level functional capacity and dental health behaviors in community-dwelling older adults. Gerodontology. 2013;30:133-40.

9. Takata Y, Ansai T, Soh I, Akifusa S, Sonoki K, Fujisawa K, et al. Relationship between chewing ability and high-level functional capacity in an 80-yearold population in Japan. Gerodontology. 2008;25:147-54.

10. Tomioka K, Kurumatani N. Hosoi H. Association between social participation and a 3-year decline in instrumental activities of daily living among community-dwelling elderly adults. J Am Geriatr Soc 2017;65:107-113..

11. Shinkai S, Kumagai S, Fujiwara Y, Amano H, Yoshida Y, Watanabe S, et al Predictors for the onset of functional decline among initially non-disabled older people living in a community during a 6-year follow-up. Geriatr Gerontol Int. 2003;3:S31-9.

12. Iwasa H, Yoshida Y, Kumagai S, Ihara K, Yoshida H, Suzuki T. Depression status as a reliable predictor of functional decline among Japanese community-dwelling older adults: a 12-year population-based prospective cohort study. Int J Geriatr Psychiatry. 2009;24:1192-200.

13. Iwasa $H$, Gondo $Y$, Yoshida $Y$, Kwon J, Inagaki H, Kawaai C, et al. Cognitive performance as a predictor of functional decline among the non-disabled elderly dwelling in a Japanese community: a 4-year population-based prospective cohort study. Arch Gerontol Geriatr. 2008;47:139-49.

14. Ohmori-Matsuda K, Kuriyama S, Hozawa A, Nakaya N, Shimazu T, Tsuji I. The joint impact of cardiovascular risk factors upon medical costs. Prev Med. 2007;44:349-55.

15. Nagai M, Kuriyama S, Kakizaki M, Ohmori-Matsuda K, Sone T, Hozawa A, et al. Impact of obesity, overweight and underweight on life expectancy and lifetime medical expenditures: the Ohsaki cohort study. BMJ Open. 2012;2: e000940.

16. Nakamura $\mathrm{K}$, Okamura $T$, Kanda H, Hayakawa $T$, Okayama A, Ueshima H, et al. Medical costs of obese Japanese: a 10-year follow-up study of National Health Insurance in Shiga. Japan Eur J Public Health. 2007;17:424-9.

17. Nagai M, Kuriyama S, Kakizaki M, Ohmori-Matsuda K, Sone T, Hozawa A, et al. Impact of walking on life expectancy and lifetime medical expenditure: the Ohsaki cohort study. BMJ Open. 2011;1:e000240.

18. Lo YT, Wahlqvist ML, Chang YH, Kao S, Lee MS. Dietary diversity predicts type of medical expenditure in elders. Am J Manag Care. 2013;19:e415-23.

19. Barnard ND, Nicholson A, Howard JL. The medical costs attributable to meat consumption. Prev Med. 1995;24:646-55.

20. Bureau S. Ministry of Internal Affairs and Communications. National population census. 2015;

21. Tsubono Y, Tsuji I, Fujita K, Nakaya N, Hozawa A, Ohkubo T, et al. Validation of walking questionnaire for population-based prospective studies in Japan: comparison with pedometer. J Epidemiol. 2002;12:305-9.

22. Kumagai S, Watanabe S, Shibata H, Amano H, Fujiwara $Y$, Shinkai S, et al. Effects of dietary variety on declines in high-level functional capacity in elderly people living in a community. Nippon Koshu Eisei Zasshi. 2003;50: 1117-24. (in Japanese)

23. Attin T, Hornecker E. Tooth brushing and oral health: how frequently and when should tooth brushing be performed? Oral Health Prev Dent. 2005;3:135-40.

24. Listl S. Oral health conditions and cognitive functioning in middle and later adulthood. BMC Oral Health. 2014;14:70.

25. OharaY HH, Watanabe $Y$, Obuchi S, Yoshida H, Fujiwara Y, et al. Factors associated with self-rated oral health among community-dwelling older Japanese: a cross-sectional study. Geriatr Gerontol Int. 2015;15:755-61.

26. Riumallo-Herl CJ, Kawachi I, Avendano M. Social capital, mental health and biomarkers in Chile: assessing the effects of social capital in a middleincome country. Soc Sci Med. 2014;105:47-58.

27. Ueshima K, Fujiwara T, Takao S, Suzuki E, Iwase T, Doi H, et al. Does social capital promote physical activity? A population-based study in Japan. PLoS One. 2010;5:e12135.

28. Uphoff EP, Pickett KE, Cabieses B, Small N, Wright J. A systematic review of the relationships between social capital and socioeconomic inequalities in health: a contribution to understanding the psychosocial pathway of health inequalities. Int J Equity Health. 2013;12:54.

29. Haley DF, Linton S, Luo R, Hunter-Jones J, Adimora AA, Wingood GM, et al. Public housing relocations and relationships of changes in neighborhood disadvantage and transportation access to unmet need for medical care. J Health Care Poor Underserved. 2017;28:315-28.

30. Inoue S, Murase N, Shimomitsu T, Ohya Y, Odagiri Y, Takamiya T, et al. Association of physical activity and neighborhood environment among Japanese adults. Prev Med. 2009;48:321-5.

31. Fukuhara S, Suzukamo Y. Manual of the SF-8 Japanese version. Institute for Health Outcomes \& Process Evaluation research: Kyoto; 2004. (in Japanese)

32. Doi Y, Minowa M, Uchiyama M, Okawa M, Kim K, Shibui $K$, et al. Psychometric assessment of subjective sleep quality using the Japanese version of the Pittsburgh sleep quality index (PSQI-J) in psychiatric disordered and control subjects. Psychiatry Res. 2000;97:165-72.

33. Hoyl MT, Alessi CA, Harker JO, Josephson KR, Pietruszka FM, Koelfgen M, et al. Development and testing of a five-item version of the geriatric depression scale. J Am Geriatr Soc. 1999;47:873-8.

34. Morris JN, Fries BE, Mehr DR, Hawes C, Phillips C, Mor V, et al. MDS cognitive performance scale. J Gerontol. 1994;49:M174-82.

35. Koyano W, Shibata H, Nakazato K, Hoga H, Suyama Y. Measurement of competence in the elderly living at home: development of an index of competence. Nippon Koshu Eisei Zasshi. 1987;34:109-14. (In Japanese)

36. Koyano W, Shibata H, Nakazato K, Haga H, Suyama Y. Measurement of competence: reliability and validity of the TMIG index of competence. Arch Gerontol Geriatr. 1991;13:103-16.

37. Fujiwara $Y$, Shinkai S, Amano H, Watanabe S, Kumagai S, Takabayashi K, et al. Test-retest variation in the Tokyo metropolitan Institute of Gerontology Index of competence in community-dwelling older people independent in daily living toward individual assessment of functional capacity. Nippon Koshu Eisei Zasshi. 2003;50:360-7. (in Japanese)

38. Tomioka K, Okamoto N, Morikawa M, Kurumatani N. Self-reported hearing loss predicts 5-year decline in higher-level functional capacity among the highfunctioning elderly: the Fujiwara-kyo study. J Am Geriatr Soc. 2015;63:2260-8.

39. Fujiwara $Y$, Shinkai S, Kumagai S, Amano H, Yoshida $Y$, Yoshida $H$, et al. Longitudinal changes in higher-level functional capacity of an older population living in a Japanese urban community. Arch Gerontol Geriatr. 2003;36:141-53.

40. Kanazawa M, Yoshiike N, Osaka T, Numba Y, Zimmet P, Inoue S. Criteria and classification of obesity in Japan and Asia-Oceania. World Rev Nutr Diet. 2005;94:1-12.

41. Barros AJ, Hirakata VN. Alternatives for logistic regression in cross-sectional studies: an empirical comparison of models that directly estimate the prevalence ratio. BMC Med Res Methodol. 2003;3:21.

42. Hoebel J, Starker A, Jordan S, Richter M, Lampert T. Determinants of health check attendance in adults: findings from the cross-sectional German health update (GEDA) study. BMC Public Health. 2014;14:913.

43. Fujiwara $Y$, Shinkai S, Kumagai S, Amano H, Yoshida Y, Yoshida H, et al. Impact of history or onset of chronic medical conditions on higher-level functional capacity among older community-dwelling Japanese adults. Geriatr Gerontol Int. 2003;3:S69-77.

44. Fujiwara Y, Watanabe S, Kumagai S, Yoshida Y, Takabayashi K, Morita M, et al. Prevalence and characteristics of older community residents with mild cognitive decline. Geriatr Gerontol Int. 2002;2:57-67.

45. Amano H, Watanabe S, Kumagai S, Yukawa H, Suzuki T, Shibata H. Glycated hemoglobin levels and intellectual activity in an aged population. J Am Geriatr Soc. 2005:53:2128-34

46. Nakamura Y, Kaneko I, Kawamura Y, Sakano T, Naito K, Maeda K, et al. Factors associated with self-rated health for non-institutionalized aged persons. Nippon Koshu Eisei Zasshi. 2002;49:409-16. (in Japanese)

47. Aida J, Kondo K, Kawachi I, Subramanian SV, Ichida Y, Hirai H, et al. Does social capital affect the incidence of functional disability in older Japanese? A prospective population-based cohort study. J Epidemiol Community Health. 2013;67:42-7.

48. Fujiwara T, Kondo K, Shirai K, Suzuki K, Kawachi I. Associations of childhood socioeconomic status and adulthood height with functional limitations among Japanese older people: results from the JAGES 2010 project. J Gerontol A Biol Sci Med Sci. 2014;69:852-9.

49. Agerholm J, Bruce D, Burström B. Comparing healthcare utilization among health survey respondents with the total population-are respondents representative? BMC Health Serv Res. 2016;16:510. 
50. Hoeymans N, Feskens EJ, Van Den Bos GA, Kromhout D. Non-response bias in a study of cardiovascular diseases, functional status and self-rated health among elderly men. Age Ageing. 1998;27:35-40.

51. Agerholm J, Bruce D, Ponce de Leon A, Burström B. Socioeconomic

differences in healthcare utilization, with and without adjustment for need: an example from Stockholm, Sweden. Scand J Public Health. 2013;41:318-25.

52. Riediger ND, Moghadasian MH. Patterns of fruit and vegetable consumption and the influence of sex, age and socio-demographic factors among Canadian elderly. J Am Coll Nutr. 2008;27:306-13.

Submit your next manuscript to BioMed Central and we will help you at every step:

- We accept pre-submission inquiries

- Our selector tool helps you to find the most relevant journal

- We provide round the clock customer support

- Convenient online submission

- Thorough peer review

- Inclusion in PubMed and all major indexing services

- Maximum visibility for your research

Submit your manuscript at www.biomedcentral.com/submit
Biomed Central 